\title{
AUTONOMIA DO PACIENTE, POLÍTICAS PÚBLICAS DE INCORPORAÇÃO DE TERAPÊUTICAS PELO SUS NA PANDEMIA DE COVID-19 E O PAPEL DA MEDICINA BASEADA EM EVIDÊNCIAS
}

\author{
PATIENT AUTONOMY, PUBLIC POLICIES FOR THE INCORPORATION OF \\ THERAPEUTICS BY THE BRAZILIAN HEALTH SYSTEM IN THE COVID-19 PANDEMIC
} AND THE ROLE OF EVIDENCE-BASED MEDICINE

\author{
Roberto Henrique Pôrto Nogueira ${ }^{1}$ \\ Universidade Federal de Ouro Preto (UFOP) \\ Felipe Melazzo do Nascimento Santos ${ }^{2}$ \\ Universidade Federal de Ouro Preto (UFOP)
}

\begin{abstract}
Resumo:
Objetiva-se analisar o papel da Medicina Baseada em Evidências no desenho da política pública relacionada à incorporação de terapêuticas pelo Sistema Único de Saúde (SUS). Para tanto, também são tangenciados os argumentos da autonomia do paciente para decidir sobre seu tratamento e da licitude da prescrição off label de medicamentos, para esclarecer a ausência de relação entre essas ideias e a adequação da disponibilização ampla de medicamentos na rede pública de saúde. Trata-se de estudo teórico-dogmático com a exploração de dados secundários na delimitação dos institutos jurídicos e conteúdos correlatos acerca da produção do conhecimento científico na seara médica. Demonstrar o papel das evidências científicas nas práticas resultantes de contextos médicos diversos mostra-se relevante para que a pandemia da COVID-19 não turve os debates sobre o direito à saúde e sobre as políticas públicas correlatas. Como resultado, tem-se que a Medicina Baseada em Evidências privilegia a relevância e a qualidade das evidências científicas para o aumento da precisão da prática terapêutica, diagnóstica e preventiva em saúde, o que constitui substrato de análise necessária para a aferição da pertinência de medidas de saúde pública.
\end{abstract}

Palavras-chave:

Autonomia. COVID-19. Medicina Baseada em Evidências. Políticas públicas.

\begin{abstract}
:
It is aimed to analyze the role of Evidence-Based Medicine in the definition of public policy related to the incorporation of therapies by the Brazilian Public Health System. To this end, the ideas of the patient's autonomy to decide on their treatment and the lawfulness of the off-label prescription of medicines are also important, in order to make it clear that there is no necessary relation between these perspectives and the adequacy of the decision on the wide availability of medicines in the public health system. It is a theoretical-dogmatic study with the exploration of secondary data in the delimitation of legal institutes and related contents about the production of scientific knowledge in the medical field. Demonstrating the role of Evidence-Based Medicine in practices resulting from different medical contexts is relevant so that the COVID-19 pandemic does not cloud the debates about the right to health and linked public policies. As a result, Evidence-Based Medicine emphasizes the relevance
\end{abstract}

\footnotetext{
${ }^{1}$ Doutor e Mestre em Direito Privado pela PUC Minas. Especialista em Direito Tributário pela Faculdade de Direito Milton Campos Belo Horizonte. Professor Adjunto IV do Mestrado Acadêmico em 'Novos Direitos, Novos Sujeitos' e da Graduação em Direito da Universidade Federal de Ouro Preto - UFOP. Pesquisador do Núcleo de Estudos Novos Direitos e Reconhecimento - NDP, do Centro de Estudos em Biodireito - CEBID-UFOP, do JUSBIOMED-UNEB e do Grupo de Apoio Jurídico à Gestão de Crise Pandêmica da COVID-19 (PPGD-UFOP). Apoiado por AUXÍLIO PESQUISADOR UFOP. Orcid: https://orcid.org/0000-0003-0216-1433. E-mail: roberto.nogueira@ufop.edu.br

${ }^{2}$ Mestrando em Direito, Área de Concentração 'Novos Direitos, Novos Sujeitos' pela Universidade Federal de Ouro Preto - UFOP. Bolsista institucional UFOP. Pesquisador vinculado aos núcleos de estudos 'Novos Direitos e Reconhecimento' - NDP - UFOP e "Centro de Estudos em Biodireito" - CEBID - UFOP. Orcid: https://orcid.org/0000-0002-9622-9293
} 
and the quality of scientific evidence for increasing the accuracy of therapeutic, diagnostic and preventive health practices, which constitutes a necessary point of reference for assessing policies with concern to public health.

Keywords:

Autonomy. COVID-19. Evidence-Based Medicine. Public policies.

\section{CONSIDERAÇÕES INICIAIS}

No panorama da pandemia da COVID-19, por que é tão importante conhecer e considerar evidências científicas no desenho da política pública relacionada à incorporação de terapêuticas pelo Sistema Único de Saúde (SUS)? A resposta a essa indagação depende da compreensão dos desafios emergentes e dos critérios de adequação da política pública em saúde, bem como da aferição da impertinência, para esse mister, dos argumentos ligados ao exercício de autonomia do paciente para decidir sobre seu tratamento e à licitude da prescrição off label de medicamentos.

A Organização Mundial da Saúde (OMS) declarou, em 30 de janeiro de 2020, a situação de emergência de saúde pública mundial decorrente da doença designada como COVID-19. Menos de dois meses depois, em 11 de março seguinte, declarou a pandemia. A transmissão do agente etiológico, qual seja, o novo Coronavírus (SARS-CoV-2, de acordo com Comitê Internacional de Taxonomia Viral), ocorre de pessoa a pessoa, independentemente de serem sintomáticas (WANG, 2020).

Nesse cenário pandêmico de uma nova doença, o Direito é desafiado a ocupar-se de problemas diversos, tais como aqueles conexos a contratações públicas, policiamento preditivo, revisão de relações jurídicas negociais privadas, responsabilidade civil do médico no âmbito da prática profissional, objeção de consciência e recusa do médico para a prescrição de medicamento. ${ }^{3}$ Para o propósito desse trabalho, desponta o problema das políticas públicas de incorporação de medicamentos pelo sistema público de saúde, em uma conjuntura na qual são inexistentes tratamentos farmacológicos específicos para a COVID-19. Assim, o tratamento regular é o sintomático, que compreende opções para o controle da febre, dor, tosse seca e náusea. Tem lugar o uso de medicações de apoio, quais sejam, antipiréticos, analgésicos, antitussígenos/expectorantes e antieméticos (BRASIL, 2020).

\footnotetext{
${ }^{3}$ Especificamente sobre a responsabilidade civil do médico no âmbito da prática profissional na pandemia da COVID-19, ver: NOGUEIRA, 2020; e LIMA; BERALDO; NOGUEIRA, 2020. O debate acerca da objeção de consciência e da recusa do médico para a prescrição de medicamento foi explorada por: NOGUEIRA; GODOI, 2020.
} 
Diante da identificação, pela comunidade científica e também pela comunidade leiga (não necessariamente ilegítima para tanto, o que se define a partir do contexto humano, social, econômico e político), de terapias alternativas não consideradas específicas para o tratamento da COVID-19, cabe compreender a relação entre a autonomia do paciente para fazer uso de uma determinada medicação, a prerrogativa do médico para prescrever off label e a fundamentação de políticas públicas de disponibilização de terapêuticas. A hipótese é a de que a Medicina Baseada em Evidências desempenha um papel central no debate.

O presente estudo apresenta-se como teórico-dogmático com a exploração de dados secundários na delimitação dos institutos jurídicos e conteúdos correlatos acerca da produção do conhecimento científico na seara médica.

Inicialmente, cabe resgatar os argumentos da autonomia do paciente na sua relação com o médico e a licitude da prescrição off label, no propósito de elucidar a provável ausência de relação entre essas ideias e a adequação da disponibilização ampla de medicamentos na rede pública de saúde.

Então, critérios legais determinantes da adequação das políticas públicas de incorporação de terapêuticas no Sistema Único de Saúde são apontados, de modo a destacar o papel da Medicina Baseada em Evidências (MBE).

Por fim, o estudo enfrenta, cientificamente, a partir dos referenciais edificados nas seções anteriores, estudos clínicos acerca da cloroquina e da hidroxicloroquina e as evidências científicas deles provenientes, para análise da consistência dos fundamentos técnicos para a incorporação e disponibilização ampla desse tratamento no âmbito do SUS. Vale dizer, o estudo não pretende discutir eficácia da medicação em apreço, mas sim demonstrar como as evidências clínicas são capitais para legitimarem essa específica política pública de saúde de incorporação de tratamento no sistema público.

Mostra-se relevante, portanto, para que o contexto da pandemia da COVID-19 não deturpe os debates sobre o direito à saúde, a demonstração do papel das evidências científicas nas políticas públicas correlatas.

\section{AUTONOMIA DO PACIENTE E PRESCRIÇÃO MÉDICA}

É comum que se recorra ao argumento da autodeterminação terapêutica, que integra o espectro da autonomia do paciente, para justificar a adoção de determinada política pública de saúde. Exatamente por essa razão, vale explorar essa compreensão, que é útil às discussões do trabalho. 
Ao paciente, cabe a prerrogativa de definir seu próprio tratamento, na edificação de sua saúde, considerada de forma ampla e holística, abrangente de seu bem-estar e delineada pelo livre desenvolvimento de sua personalidade. Isso viabiliza a sua participação, como protagonista, na relação médico-paciente, contexto no qual deve ser definida, de modo dialógico, a abordagem terapêutica. Caso não haja objeção de consciência lícita do médico, e desde que esclarecimentos tenham sido prestados em relação às indicações, às contraindicações, aos efeitos adversos e colaterais e ao estado da arte do conhecimento médico, o paciente preserva a prerrogativa de eleger ou de rejeitar as terapias disponíveis. O exercício efetivo e idôneo de sua autonomia guarda relação direta com a responsabilidade correlata. Independentemente da relação médico-paciente, a opção pelo uso do medicamento pelo paciente é ato de autonomia, desde que se trate de produto de acesso permitido, nos moldes dos requisitos fixados pelo ordenamento jurídico e pela vigilância sanitária.

Ao médico, lado outro, cabe prescrever tratamentos. Prescrever, do latim praescribere, significa ordenar, de maneira explícita, previamente; indicar com precisão; determinar, fixar, preceituar; indicar como remédio; receitar, indicar (FERREIRA, 2004). No Brasil, prescrever medicamentos é dever profissional do médico (BRASIL, 1932). Outros profissionais de saúde, nos limites legalmente previstos, podem prescrever medicamentos (NOGUEIRA, 2017, p. 60).

Para prescrever, o Código de Ética Médica (2018) veda ao médico deixar de obter consentimento do paciente ou de seu representante legal após esclarecê-lo sobre o procedimento a ser realizado, salvo em caso de risco iminente de morte.

Na mesma linha, para o médico fazer objeção ao desejo do paciente e recusar-se a prescrever determinado tratamento, não deve fundamentar-se no fato ou ato que padeça de civilidade ou de consideração e respeito pela dignidade do paciente, sendo vedada a discriminação. No geral, portanto, o médico deve aceitar as escolhas dos pacientes. Essas orientações deontológicas reforçam a autonomia do paciente, para promoverem oportunidades e condições de exercício do direito de decidir livremente sobre sua pessoa e sobre seu bemestar.

Cabe, portanto, ao médico, prescrever a terapêutica adequada quando seu uso estiver liberado no País. A liberação, pela via do registro para autorização de produção ou de comercialização de medicamento no Brasil, é ato do Ministério da Saúde, sob responsabilidade da Agência Nacional de Vigilância Sanitária (ANVISA). O médico pode, ainda, prescrever terapêutica experimental, se efetivamente aceita pelos órgãos competentes e com o consentimento esclarecido do paciente. 


\section{O REGISTRO E A PRESCRIÇÃO OFF LABEL DE MEDICAMENTOS}

A Lei n. 6.360 (BRASIL, 1976) almeja, com o registro do medicamento junto à ANVISA, possibilita o controle de qualidade, por meio de um conjunto de medidas assecuratórias da pureza, da eficácia e da inocuidade. A Resolução da Diretoria Colegiada da ANVISA RDC n. 200 (AGÊNCIA NACIONAL DE VIGILÂNCIA SANITÁRIA, 2017), para a concessão de registro a um determinado medicamento novo, exige que estudos clínicos prévios sejam desenvolvidos sob exigências específicas. Logo, nos exatos termos das pesquisas implementadas, o medicamento é registrado. Se o medicamento obtém êxito, nas pesquisas devidamente controladas e nas fases consideradas mínimas, quanto aos requisitos de segurança, eficácia e acurácia, para uma doença definida, em certa população, dosagem e modo de uso, a prescrição on label se restringe a esses parâmetros, apenas. A prescrição estranha a essas diretrizes registradas no órgão de vigilância nacional é off label.

Ocorre que, havido o registro, o medicamento fica disponível para acesso. Logo, o médico pode prescrevê-lo, licitamente, mesmo fora daqueles critérios exatos registrados (prescrição off label), em vista da situação e dos interesses circunscritos e individuais do paciente. Para tanto, a prescrição off label pode ser lícita em casos extremos, de inexistência de tratamentos previamente testados, de não resposta do doente a terapias consagradas, de superioridade de tratamento inovador, de evidências científicas robustas e de ponderação do interesse do paciente (formatado com a sua participação esclarecida e ampla) (NOGUEIRA, 2017, p. 62).

Significa que mesmo que o paciente queira fazer uso off label de um medicamento, o médico pode, por objeção de consciência, não prescrevê-lo. Lado outro, mesmo que o médico deseje prescrever determinado medicamento off label, não significa que o paciente fará o uso, eis que a sua autonomia deve ser respeitada. E mais: mesmo que a evidência científica seja de baixo grau, no caso individualizado de um paciente, pode haver motivos suficientes à prescrição de uma terapia off label.

A Resolução da Diretoria Colegiada da ANVISA RDC n. 9 (AGÊNCIA NACIONAL DE VIGILÂNCIA SANITÁRIA, 2015) é responsável por definir os procedimentos e requisitos para realização de ensaios clínicos feitos no Brasil com medicamentos a serem aprovados pela agência. Tais ensaios devem seguir a Resolução quando envolvem nova indicação terapêutica, nova via de administração, nova concentração, nova forma farmacêutica, ampliação do uso, nova posologia, novas associações ou qualquer alteração pós-registro, incluindo renovação. 
O estudo clínico, para que esteja em consonância com a normativa em questão, deve, necessariamente, estar acompanhado de um Dossiê de Desenvolvimento Clínico de Medicamento (DDCM), que consiste em compilado de documentos para submissão à ANVISA, com finalidade de permitir que sejam avaliadas todas as etapas inerentes ao experimento projetado. Tal documentação deve possibilitar a avaliação de eficácia e de segurança, tanto no que respeita aos direitos dos participantes em todas as fases do estudo, quanto à qualidade do medicamento até então experimental. Ressalta-se que, uma vez cancelado um DDCM, seja por justificativas técnico-científicas dos pesquisadores, seja por motivos de segurança, nenhum ensaio clínico que carregue alguma relação com ele poderá ser continuado no País (AGÊNCIA NACIONAL DE VIGILÂNCIA SANITÁRIA, 2015).

A Resolução exige também que os estudos clínicos sigam as Boas Práticas Clínicas (BPC), que envolvem:

[...] a definição de um padrão para o planejamento, a condução, a realização, o monitoramento, a auditoria, o registro, a análise e o relato de ensaios clínicos que fornece a garantia de que os dados e os resultados relatados têm credibilidade e precisão, e que os direitos, a integridade e o sigilo dos participantes do ensaio clínico estão protegidos, de acordo com as diretrizes de BPC dispostas no Documento das Américas e Manual de Boas Práticas Clínicas da Conferência Internacional de Harmonização (AGÊNCIA NACIONAL DE VIGILÂNCIA SANITÁRIA, 2015).

Um estudo clínico cujas fases estejam em consonância com esse regime regulamentar revela-se científico do ponto de vista do ente regulador e, assim, pretende engendrar evidências científicas consideradas robustas para autorizar o registro junto à autoridade sanitária. Com efeito, a preocupação institucional em regular os estudos clínicos que afiram a segurança e a eficácia de medicamentos revela o reconhecimento institucional da relevância das evidências clínicas no contexto das políticas públicas.

\section{POLÍTICAS PÚBLICAS DE INCORPORAÇÃO TERAPÊUTICA NO ÂMBITO DO SISTEMA ÚNICO DE SAÚDE E A MEDICINA BASEADA EM EVIDÊNCIAS}

De todo modo, esses argumentos da autodeterminação terapêutica do paciente e da licitude circunstancial da prescrição off label de medicamentos parecem ter pouco ou nada a ver com a opção por uma dada política pública de incorporação e de disponibilização ampla de determinados tratamentos no SUS.

Tratando-se de uma política pública que envolve gastos significativos e uma abordagem transversal no sistema de saúde para a população em geral, o interesse coletivo há de ser 
considerado. Para a eleição dessa política pública, deve haver, portanto, atendimento a critérios intersubjetivos firmados por processos democraticamente legitimados.

No Brasil, a Lei n. 8.080 (BRASIL, 1990) introduz esses critérios objetivos, de modo a demandar que a incorporação, a exclusão ou a alteração pelo SUS de novos medicamentos, produtos e procedimentos, bem como a constituição ou a alteração de protocolo clínico ou de diretriz terapêutica, sejam objeto de relatório da Comissão Nacional de Incorporação de Tecnologias, que deve levar em consideração, necessariamente, entre outros aspectos, as evidências científicas sobre a eficácia, a acurácia, a efetividade e a segurança.

Assim, independentemente de qualquer problematização acerca da epistemologia possivelmente pouco plural em relação ao critério objetivo para a decisão pela execução de uma política pública de amplo espectro na área da saúde, tal critério é, a priori, legitimamente demarcado pela lei, de maneira que parece imprescindir, de modo ideal e conforme a normativa de regência, de evidências científicas em razoável grau de consistência.

As evidências clínicas científicas são resultantes de processos de produção e de revisão de conhecimentos, preferencialmente oriundos de achados em pesquisas clínicas metodologicamente estruturadas e que, devidamente divulgadas, possam contribuir para o estabelecimento de parâmetros e de práticas úteis à realização de escolhas diagnósticas, terapêuticas e profiláticas com algum grau de certeza e de segurança. Vale dizer, para diagnóstico médico, as evidências podem sugerir protocolos ou abordagens que envolvem os formatos das consultas e dos exames clínicos, que se delineiam desde a anamnese até a coleta de dados próprios do histórico do paciente, com a identificação de sintomas e para a definição do quadro clínico de uma possível doença. A depender da necessidade e da conveniência para o paciente, as evidências científicas podem precisar métodos, técnicas e drogas com superiores segurança, eficácia e acurácia para a prescrição e para a realização de tratamentos. Do mesmo modo, as evidências científicas podem contribuir para a adoção de medidas efetivamente úteis na profilaxia e na atenuação de doenças.

A Medicina Baseada em Evidências (MBE) é um movimento formado por cientistas, médicos e profissionais da saúde envolvidos e comprometidos com a utilização e com o desenvolvimento de métodos e de abordagens rigorosos que ofereçam respostas a questões clínicas “sobre eficácia, efetividade, eficiência e segurança de determinado tratamento e prevenção, bem como sobre a sensibilidade e especificidade de testes diagnósticos e aspectos prognósticos de certa doença na área da saúde” (EL DIB, 2014, p. 09).

Não somente atrelada à relação médico-paciente, a MBE também consiste na utilização da Epidemiologia Clínica como ferramenta de apoio à tomada de decisão coletiva. Logo, a área 
de estudo fornece subsídios científicos para a tomada de decisões não só de cientistas, de profissionais da saúde e de pacientes, mas também de gestores públicos de saúde no cumprimento de suas atribuições (MINISTÉRIO DA SAÚDE, 2014, p. 17).

A MBE almeja, portanto, contribuir para a redução da "margem de erro dos atos médicos com medidas custo-efetivas. O processo de aplicação da MBE pressupõe conceitos da Epidemiologia Clínica aliados a conceitos da Estatística e da Informática Médica” (GOMES, 2001, p. 4). Isso não quer dizer que a autonomia do paciente e o seu quadro clínico individual sejam ignorados, pois é a partir do paciente que "são construídas as questões clínicas a serem respondidas, e para ele, individualmente, os resultados serão aplicados, se forem válidos, importantes e úteis.” (GOMES, 2001, p. 11).

A qualidade das evidências depende da consistência dos resultados e das conclusões de seus estudos de base. Essa consistência submete-se, a seu turno, à validade interna (estrutura metodológica) e à validade externa (possibilidade de generalização dos resultados para outras populações) (GOMES, 2001, p. 17).

Logo, é o desenho dos estudos que determina a qualidade das evidências. As evidências categorizadas como de alto grau de consistência são consideradas boas para apoiar a recomendação de um determinado uso de medicamento. A depender dos fins da investigação, evidências de alto grau são aquelas apresentadas por um ou mais estudos com adequada e controlada metodologia, “prospectivos, randomizados, mascarados e controlados” (GOMES, 2001, p. 34).

O estudo é prospectivo quando é desenhado no presente e acompanhado para o futuro. É randomizado quando, em estudos experimentais, há aleatoriedade na distribuição da intervenção em relação aos participantes da pesquisa, individualmente considerados. É mascarado ou cego quando for desconhecido se o participante da pesquisa integra o grupo de intervenção ou o grupo controle. Caso o pesquisador também impeça seu próprio acesso a essa informação no curso da investigação, diz-se que o estudo é duplo cego. Quando variáveis que possam impactar os desfechos são acompanhadas e, eventualmente, isoladas, diz-se que o estudo é controlado.

Na proporção em que estudos, a depender de seus escopos, distanciam-se dessas características, eles fragilizam suas validades interna ou externa, de forma a restar minorada a qualidade das evidências, que caem de grau.

Nesse mesmo sentido, estudos com populações inadequadamente reduzidas e meros relatos de casos isolados são tidos como geradores de evidências menos robustas. 
Opiniões de perito sem avaliação crítica e pesquisas desenvolvidas apenas em laboratório tendem a produzir evidências de ainda menor consistência (GOMES, 2001, p. 34).

As evidências, por conseguinte, devem ser percebidas em graus de consistência.

No âmbito da MBE, sistemas são elaborados para a avaliação de graus de qualidade ou de consistência, bem como de graduação da força de recomendação de evidências científicas obtidas nos estudos clínicos. Esses sistemas objetivam, respectivamente, informar a confiança nas evidências apresentadas e a ênfase para que determinada conduta seja adotada ou rejeitada.

Dos marcos teóricos disponíveis que estabelecem critérios de avaliação de evidências, o presente estudo foca no Sistema GRADE (Grading of Recommendations Assessment, Development and Evaluation), tendo em vista a sua adoção pelo Ministério da Saúde. O Sistema GRADE, utilizado também pela Organização Mundial da Saúde (OMS), tem como objetivo criar um sistema universal, "transparente e sensível para graduar a qualidade das evidências e a força das recomendações” (MINISTÉRIO DA SAÚDE, 2014, p. 18).

No Sistema GRADE, os níveis ou qualidades das evidências são divididos em alto, moderado, baixo e muito baixo. Oferecem evidências com nível alto na escala do Sistema aqueles estudos clínicos bem delineados, randomizados, com amostra representativa da população, grupos paralelos, com controles de variáveis estranhas e análise de dados e achados consistentes (MINISTÉRIO DA SAÚDE, 2014, p. 45). Em alguns casos, estudos observacionais podem oferecer evidências de alto nível, desde que bem delineados e que apresentem achados consistentes, como os estudos de coorte prospectivos e conduzidos por meio de métodos especiais de análise que controlam variáveis de confusão e mostram efeitos das intervenções terapêuticas que não podem ser resultados de vieses (VILLAS BOAS; DO VALLE, 2014, p. 103). Quando o nível é alto, há forte confiança de que o verdadeiro efeito esteja muito próximo daquele obtido pelo estudo e é improvável que futuros trabalhos modifiquem tal confiança (MINISTÉRIO DA SAÚDE, 2014, p. 45).

Oferecem evidência com nível moderado os ensaios clínicos com limitações leves (problemas na condução, inconsistência nos resultados, desfechos substitutos ou validade externa comprometida), estudos observacionais com achados e estudos meramente observacionais, mas bem delineados e com achados consistentes. Em evidências de nível moderado há a confiança moderada de que os efeitos obtidos nos ensaios representam os reais. Dessa forma, trabalhos futuros são esperados a modificar tal confiança, na medida em que podem se valer de métodos mais robustos aptos a provarem outros efeitos e estimativas (MINISTÉRIO DA SAÚDE, 2014, p. 45). 
Evidências de nível baixo são obtidas a partir de estudos observacionais com importantes limitações e suscetíveis a inúmeros vieses, como os observacionais comparativos (coorte e caso-controle). A confiança nas evidências de nível baixo é limitada, visto que trabalhos futuros com melhores estruturas provavelmente impactarão na estimativa de efeito obtida (MINISTÉRIO DA SAÚDE, 2014, p. 45).

Estudos clínicos com limitações graves (com vieses no delineamento do estudo, nãocontrolados no que toca às variáveis estranhas e não sistematizados), estudos meramente observacionais também com limitações graves, estudos observacionais não comparados (como séries e relatos de caso) e opiniões de especialistas oferecem evidências de nível muito baixo. Nessas circunstâncias, qualquer estimativa obtida pelos estudos é incerta, uma vez que as fortes limitações metodológicas interferem diretamente na confiança dos resultados obtidos (MINISTÉRIO DA SAÚDE, 2014, p. 45).

Dessa forma, os ensaios clínicos randomizados são os aptos a oferecerem evidências de nível alto no Sistema GRADE. Quando são considerados apenas estudos observacionais, o nível de evidência inicia-se baixo. Além desse, outros fatores são responsáveis pela redução do nível de qualidade da evidência, como limitações metodológicas, inconsistências, uso de evidências indiretas, imprecisão e viés de publicação (MINISTÉRIO DA SAÚDE, 2014, p. 19).

As limitações metodológicas estão diretamente relacionadas com o alto potencial de presença de vieses nos estudos, vale dizer, quanto maior a limitação, maiores serão os vieses. Com base na análise das características do experimento, o Sistema GRADE organizou e estruturou uma forma de avaliação do Risco de Viés (ou RoB, Risk of Bias), que leva em consideração suas limitações metodológicas referentes ao seu delineamento ou execução (MINISTÉRIO DA SAÚDE, 2014, p. 23). O Risco de Viés também apresenta graus ou níveis: alto, baixo ou incerto (VILLAS BOAS; DO VALLE, 2014, p. 27).

São exemplos de limitações metodológicas de estudos randomizados: ausência de sigilo da alocação, ausência de mascaramento, seguimento incompleto (perda no seguimento de pacientes randomizados e ausência de análise por intenção de tratar), relato seletivo de desfechos e outros entraves, como interrupção precoce do estudo por benefício e utilização de desfechos não validados (VILLAS BOAS; DO VALLE, 2014, p. 47).

Em relação aos estudos observacionais, são exemplos de limitações metodológicas: seleção e inclusão inadequada de participantes, ausência de mascaramento, falhas para controlar adequadamente os fatores de confusão e seguimento incompleto (VILLAS BOAS; DO VALLE, 2014, p. 47). 
Além dos níveis de evidência, o Sistema GRADE também estipula a chamada força de recomendação, que expressa a ênfase para que seja adotada ou rejeitada determinada conduta de saúde por gestores públicos de saúde, pacientes e profissionais, considerando potenciais vantagens e desvantagens obtidas em estudos. A força de recomendação é medida em forte ou fraca (MINISTÉRIO DA SAÚDE, 2014, p. 20). Percebe-se que o nível das evidências obtidas nos estudos clínicos é fator que influencia a força de recomendação.

Como demonstrado, com base nas disposições da Lei 8.080 e na adoção do Sistema GRADE pelo Ministério da Saúde, tem-se que a Medicina ou Saúde Baseada em Evidências ocupa uma posição central não apenas nas situações próprias da relação médico-paciente (autonomia do paciente, prescrição de medicamentos), mas também na tomada de decisão dos agentes públicos e na fundamentação de políticas públicas de disponibilização de terapêuticas.

\section{TRATAMENTOS INCORPORADOS PELO SUS NA PANDEMIA DE COVID-19}

A ausência de tratamento farmacológico específico para a COVID-19 favorece o emprego de medicamentos já conhecidos e até então utilizados no tratamento de outras enfermidades, no controle da doença em estudo. É o caso do difosfato de cloroquina ou somente cloroquina e seu análogo sulfato de hidroxicloroquina ou somente hidroxicloroquina, manipulados, originalmente, no tratamento de malária e de doenças autoimunes como lúpus eritematoso sistêmico e artrite reumatóide (ORGANIZAÇÃO PAN-AMERICANA DA SAÚDE, 2020, p. 2). Estudos clínicos, portanto, dedicam-se ao teste da eficácia de drogas voltadas a outros fins, nas diversas abordagens da COVID-19.

Vale frisar, os estudos clínicos podem oferecer evidências científicas aptas a comporem o substrato para a identificação de terapias principais e adjuvantes para a COVID-19, para prescrição médica, para o uso pelo paciente e, eventualmente, para a incorporação ampla pelo sistema de saúde (ORGANIZAÇÃO PAN-AMERICANA DA SAÚDE, 2020, 2020, p. 2).

Com o intuito de descortinar o papel da MBE e das evidências científicas propriamente ditas para a legitimidade das políticas públicas de incorporação e de disponibilização de terapias no âmbito da saúde, é válido o esforço de análise dos estudos clínicos recorrentemente aludidos como fundamentos para a admissão ampla da cloroquina e da hidroxicloroquina pelo SUS, bem como o diálogo com as posições do Conselho Federal de Medicina (CFM) e da Sociedade Brasileira de Infectologia emitidas no decorrer do período compreendido entre março e julho de 2020. 
Em 06 de março de 2020 foi publicado na revista Journal of ZheJiang University (Medical Sciences) um estudo chinês realizado no Shanghai Public Health Clinical Center, cujo objetivo era avaliar a eficácia e a segurança do uso da hidroxicloroquina (HCQ) no tratamento de pacientes com COVID-19 considerada moderada (CHEN et al., 2020, p. 215).

Tratou-se de um estudo clínico randomizado (ECR), duplo-cego, prospectivo, com uma amostra de 30 pacientes com idade média de 48,5 anos diagnosticados com COVID-19, matriculados mediante consentimento informado e cujo critério de inclusão era não ter recebido nenhum outro tratamento para a doença até o início do estudo. Os pacientes foram divididos em dois grupos, um controle contendo 15 pessoas e outro experimental também com 15 (CHEN et al., 2020, p. 216).

Durante 5 dias, o grupo controle recebeu tratamento convencional (inalação com oxigênio, medicamentos antivirais recomendados no plano de tratamento, nebulização e antibacterianos, quando necessários), enquanto o grupo experimental recebeu 400mg de hidroxicloroquina por dia aliada ao tratamento convencional (CHEN et al., 2020, p. 217).

Ao final do experimento, 13 (86,7\%) pacientes do grupo experimental e 14 (93,3\%) pacientes do grupo controle testaram negativo para COVID-19; a duração média de hospitalização até os resultados negativos foi de 4 dias para o grupo experimental e 2 dias para o grupo controle; o tempo médio para a normalização da temperatura corporal dos pacientes, após a hospitalização, foi igual no grupo controle e experimental, isto é, 1 dia; houve progressão radiológica, constatada por imagens de tomografia computadorizada, em 5 (33,3\%) casos no grupo experimental e em 7 (46,7\%) casos no grupo controle; 4 casos (26,7\%) do grupo experimental e 3 casos (20\%) do grupo controle apresentaram diarreia transitória e função hepática anormal (CHEN et al., 2020, p. 218).

A partir desses resultados, os pesquisadores concluíram que o prognóstico para pacientes moderados de COVID-19 era bom. Entretanto, sugeriram a necessidade de estudos maiores em relação ao tamanho da amostra para identificar os efeitos da hidroxicloroquina no tratamento (CHEN et al., 2020, p. 218).

Com base nos resultados apresentados, percebe-se que, se comparada ao grupo controle, a dose padrão de hidroxicloroquina não apresentou efeitos clínicos consideráveis na melhora dos sintomas e na aceleração da supressão do SARS-CoV-2 nos pacientes, na forma aferida.

A Organização Pan-Americana da Saúde (OPAS), vinculada a Organização Mundial da Saúde (OMS), divulgou em 22 de abril de 2020 uma revisão rápida do documento de “Atualização Dinâmica e Contínua de Potenciais Terapias para COVID-19: resumo de revisões sistemáticas rápidas”. O documento realizou um agrupamento meta-analítico de diversos 
estudos, realizados até o momento da publicação, de desenvolvimento e/ou aproveitamento de medicamentos que possam ter efeito contra o SARS-CoV-2.

Segundo o documento (ORGANIZAÇÃO PAN-AMERICANA DA SAÚDE, 2020, p. 9), o estudo realizado no Shanghai Public Health Clinical Center apresentou um Risco de viés alto (ou RoB, Risk of Bias), definido com base no Sistema GRADE, e um Nível de certeza de evidência muito baixo, por (i) trabalhar com uma amostragem pequena com poucos eventos clínicos, logo, não poderia ser um estudo representativo; e por (ii) atribuir co-tratamento de forma não balanceada.

A atribuição de co-tratamento não balanceada mencionada pela OPAS é resultado do controle não realizado pelo estudo chinês acerca do tratamento convencional manipulado contemporaneamente em ambos os grupos, no decorrer do experimento. Não há menções especificas em relação à dosagem, ao momento de administração e a quais pacientes tal tratamento foi necessário. O co-tratamento ou tratamento convencional, se não controlados, podem funcionar como variáveis aptas a macular os resultados do estudo.

Em 20 de março de 2020, foi publicado um estudo francês na revista International Journal of Antimicrobial Agents, coordenado no âmbito de The Méditerranée Infection University Hospital Institute in Marseille. O objetivo do estudo era avaliar se a cloroquina e a hidroxicloroquina eram eficazes no combate do SARS-CoV-2 (GAUTRET et al., 2020, p. 2).

Tratou-se de um estudo observacional clínico, não-randomizado, não mascarado, prospectivo, com uma amostra de 36 pacientes com idade média de 45,1 anos, hospitalizados, diagnosticados com COVID-19 por meio do exame PCR e em estados clínicos diversos. Os pacientes foram divididos em um grupo controle de 16 pessoas e um grupo experimental de 20 pessoas (GAUTRET et al., 2020, p. 2-3).

Foram excluídas gestantes e mulheres em período de amamentação, bem como pacientes que tinham alergia conhecida à hidroxicloroquina ou à cloroquina ou que tinham contraindicação aos medicamentos, incluindo retinopatia, deficiência de glicose-6-fostato desidrogenase (G6PD) e prolongamento do intervalo QT. Como o estudo não foi randomizado, esses pacientes diagnosticados com COVID-19 e excluídos por não preencherem os critérios de inclusão, juntamente com crianças e com aqueles que se recusaram a receber o tratamento da hidroxicloroquina ou da cloroquina, formaram o grupo controle (GAUTRET et al., 2020, p. 2).

A amostra, para fins de análise dos resultados, foi dividida também em 3 categorias: pacientes assintomáticos (2 estavam no grupo experimental e 4 no grupo controle); pacientes com sintomas de infecção respiratória do trato superior ou upper respiratory tract infection 
(URTI), quando apresentavam rinite, faringite ou febre alta e mialgia isolada de baixo grau (12 estavam no grupo experimental e 10 estavam no grupo controle); e pacientes com sintomas de infecção no trato inferior ou lower respiratory tract infections (LRTI), quando apresentavam sintomas de pneumonia ou bronquite (6 estavam no grupo experimental e 2 no grupo controle) (GAUTRET et al., 2020, p. 2).

Durante 6 dias, o grupo experimental recebeu 200mg de hidroxicloroquina por via oral, 3 vezes ao dia e, a depender do estado clínico, também recebeu azitromicina. Do grupo experimental, 14 pacientes foram tratados apenas com hidroxicloroquina e 6 foram tratados com hidroxicloroquina aliada a azitromicina. Em relação ao grupo controle, o estudo apenas mencionou que o tratamento sintomático e a administração de antibióticos, como medidas de prevenção de superinfecção bacteriana, foram implementados conforme julgamento clínico. (GAUTRET et al., 2020, p. 2-4). Não há, assim, como afirmar que o mesmo tratamento, ainda que sintomático, foi aplicado a todos os pacientes do mesmo grupo.

No final do experimento, dos 14 pacientes que receberam apenas hidroxicloroquina, 8 (57,1\%) testaram negativo para COVID-19; dos 6 pacientes que receberam hidroxicloroquina aliada a azitromicina, 6 (100\%) testaram negativo para COVID-19; e dos 16 pacientes presentes no grupo controle, 2 (12,5\%) testaram negativo para COVID-19. Analisando os dados do grupo experimental de outra forma, 14 (70\%) dos 20 pacientes que receberam hidroxicloroquina, seja ela aliada a azitromicina ou não, testaram negativo no final do experimento (GAUTRET et al., 2020, p. 4).

Após a apresentação dos resultados, os pesquisadores concluíram que, apesar da amostra conter um número pequeno de pacientes, o estudo demonstrou que a hidroxicloroquina foi eficiente no combate do agente etiológico SARS-Cov-2 em pacientes com COVID-19 em um curto espaço de tempo (3 a 6 dias), no formato de aferição adotado pelo estudo (redução da detecção do vírus no trato respiratório superior). Além disso, concluíram que tal efeito do medicamento é reforçado quando aliado a azitromicina (GAUTRET et al., 2020, p. 5).

Segundo a revisão rápida de 22 de abril de 2020 do documento “Atualização Dinâmica e Contínua de Potenciais Terapias para COVID-19: resumo de revisões sistemáticas rápidas”, elaborado pela Organização Pan-Americana da Saúde (OPAS), o estudo realizado no The Méditerranée Infection University Hospital Institute in Marseille apresentou um Risco alto de viés (ou RoB, Risk of Bias), definido com base no Sistema GRADE, e um Nível de certeza de evidência muito baixo, por (i) não discutir a ocorrência de efeitos colaterais dos medicamentos manipulados; (ii) conter a presença de confundidores (variáveis estranhas); (iii) não ser mascarado; (iv) conter amostra pequena (v) registrar poucos eventos; e (vi) conter relato 
subótimo de métodos e desfechos (ORGANIZAÇÃO PAN-AMERICANA DA SAÚDE, 2020, p. 9).

A presença de confundidores e o relato subótimo de métodos e desfechos mencionados pela OPAS dão-se pelo fato de não haver no estudo maiores descrições acerca da condição dos participantes do experimento em relação à coexistência de outras doenças, do recebimento ou não algum tipo de tratamento anterior ao experimento, bem como de outras variáveis que, se isoladas ou ao menos consideradas, poderiam alterar o resultado dos tratamentos.

No dia 01 de abril de 2020 foi publicada a Nota Informativa $n^{\circ}$ 6/2020-DAF/SCTIE/MS (BRASIL, 2020) pelo Departamento de Assistência Farmacêutica e Insumos Estratégicos da Secretaria de Ciência, Tecnologia, Inovação e Insumos Estratégicos em Saúde do Ministério da Saúde. A Nota, com base na Lei nº 13.979 (BRASIL, 2020), informa a disponibilização da cloroquina para uso no Sistema Único de Saúde, em casos confirmados e a critério médico, de pacientes hospitalizados com formas graves de COVID-19, sem que outras medidas fossem desprezadas (BRASIL, 2020).

Segundo a Nota, a justificativa para a disponibilização foi de que até aquele momento não existia disponível outro tratamento específico e eficaz, além de haver dezenas de estudos clínicos nacionais e internacionais destinados a mensurar a eficácia e a segurança da droga cloroquina (e seu análogo hidroxicloroquina) para o tratamento da infecção por SARS-Cov-2 (BRASIL, 2020).

Foi ressaltado na Nota que, a depender de novas evidências científicas que porventura viessem a surgir desses estudos, a medida poderia ser modificada a qualquer momento (BRASIL, 2020). Como exemplo desses estudos internacionais mencionados, foi referenciado o estudo francês havido pelo The Méditerranée Infection University Hospital Institute in Marseille e atestado pela Organização Pan-Americana da Saúde (OPAS) com o Risco de viés (ou RoB, Risk of Bias) alto e um Nível de certeza de evidência muito baixo, devido as suas falhas metodológicas (ORGANIZAÇÃO PAN-AMERICANA DA SAÚDE, 2020, p. 11).

Em 20 de maio de 2020, uma nova Nota Informativa foi publicada, dessa vez pelo Gabinete da Secretaria Executiva do Ministério da Saúde, e que recebeu o no 9/2020SE/GAB/SE/MS. A Nota informou a orientação permissiva e ampliativa para o tratamento com hidroxicloroquina e cloroquina, associadas ou não a azitromicina, a pacientes diagnosticados com COVID-19 no SUS, com sintomas leves e moderados, incluindo o acesso precoce. A Nota trouxe também orientações e algoritmos para o tratamento (BRASIL, 2020).

Na introdução da Nota, especificamente no ponto 2, foi disposto que o acompanhamento dos resultados dos estudos promovidos pela comunidade científica acerca dos possíveis 
tratamentos para a COVID-19 foi de extrema relevância para a manutenção e para a atualização periódica das orientações para o trato da doença (BRASIL, 2020).

Ambos os estudos comentados acima, quais sejam, o chinês e o francês, a despeito de suas falhas metodológicas (ORGANIZAÇÃO PAN-AMERICANA DA SAÚDE, 2020, p. 11), foram referenciados na Nota Informativa nº 9/2020-SE/GAB/SE/MS (BRASIL, 2020).

Ainda na introdução, dessa vez no ponto 13, a Nota justificou a ampliação da disponibilização dos medicamentos em questão, a partir do Processo-Consulta CFM $n^{\circ}$ 8/Parecer CFM n 4/2020 do Conselho Federal de Medicina (CFM) divulgado em 16 de abril (BRASIL, 2020). Tal parecer propôs a consideração do uso da cloroquina e da hidroxicloroquina no tratamento de pacientes com sintomas leves no início do quadro clínico, pacientes com sintomas importantes, mas que ainda não haja a necessidade de cuidados intensivos, e pacientes críticos recebendo cuidados intensivos, sempre sob consentimento livre e esclarecido do paciente ou dos familiares, quando cabível; sob orientação da autonomia do médico; e condicionada à valorização da relação médico-paciente.

Na data de 22 de maio, foi publicado pela revista inglesa The Lancet o estudo "Hydroxychloroquine or chloroquine with or without a macrolide for treatment of COVID-19: a multinational registry analysis”, que objetivou analisar a eficácia da cloroquina e da hidroxicloroquina associadas ou não a um macrolídeo de segunda geração (azitromicina ou claritromicina) no tratamento da COVID-19 (MEHRA; DESAI; RUCHITZKA; PATEL, 2020, p. 1).

O estudo não foi randomizado e não foi mascarado, visto que se tratou de uma pesquisa ex-post-facto (retrospectiva), isto é, não houve manipulação de tratamento pelos pesquisadores (SELLTIZ; WRIGHTSMAN; COOK; KIDDER, 1987). Os resultados foram obtidos depois que as variáveis (cloroquina e hidroxicloroquina associadas ou não a um macrolídeo de segunda geração no tratamento da COVID-19) em questão já tinham incidido no cotidiano médico.

Foi realizada uma análise multinacional dos registros de 671 hospitais localizados na América do Norte, Europa, Ásia, África, América do Sul e Austrália, disponibilizados pela Surgisphere Corporation. Esses registros continham dados não identificados de pacientes hospitalizados com COVID-19 de dezembro de 2019 a abril de 2020 e foram obtidos por meio da extração automática de registros eletrônicos de saúde hospitalares e ambulatoriais, bancos de dados da cadeia de suprimentos e registros financeiros (MEHRA; DESAI; RUCHITZKA; PATEL, 2020, p. 2).

Os dados de 96.032 pacientes foram delimitados como a amostra do estudo e divididos em 5 grupos. Os pacientes que iniciaram o tratamento dentro de 48 horas após o diagnóstico, 
com cloroquina isolada, cloroquina com um macrolídeo, hidroxicloroquina isolada ou hidroxicloroquina com macrolídeo formaram o grupo experimental, totalizando 14.888 pacientes. Os pacientes que não receberam o tratamento com os medicamentos formaram o grupo controle, totalizado por 81.444 pacientes (MEHRA; DESAI; RUCHITZKA; PATEL, 2020, p. 3).

Com base na análise dos dados primários desses pacientes, o estudo concluiu que o uso da hidroxicloroquina ou cloroquina (com ou sem macrolídeo) não apresentou benefício (nos termos aferidos no projeto investigativo), mas foi associado com um aumento no risco de arritmias ventriculares e com um maior risco de morte hospitalar por COVID-19. Além disso, o estudo sugeriu que os medicamentos não fossem empregados fora do contexto de pesquisas científicas, chamando atenção para a necessidade de confirmação urgente, com base em ensaios clínicos randomizados, da possibilidade de sua utilização (MEHRA; DESAI; RUCHITZKA; PATEL, 2020, p. 7-8).

Entretanto, no dia 05 de junho de 2020, o mesmo estudo foi retratado pela revista The Lancet, devido a preocupações acerca da veracidade dos dados disponibilizados e a análises conduzidas pela Surgisphere Corporation. Para a verificação das suspeitas, a revista lançou mão de uma revisão independente por pares para confirmar a integridade do banco de dados utilizados no artigo (MEHRA; RUCHITZKA; PATEL, 2020, p. 1820).

Na retratação, foi informado ainda que a Surgisphere não transferiu aos responsáveis pela revisão os dados, os contratos dos clientes e o relatório de auditoria completos, pois, essa transferência violaria os requisitos de confidencialidade presente nas avenças. Dessa forma, como a revisão por pares se fez prejudicada, os autores solicitaram a retirada do artigo, visto que a veracidade das fontes de dados primárias não pôde ser garantida (MEHRA; RUCHITZKA; PATEL, 2020, p. 1820).

Na data de 16 de julho de 2020 mais um estudo, cujo objetivo era investigar se a hidroxicloroquina seria capaz de reduzir a carga viral de pacientes adultos contaminados com SARS-Cov-2, foi publicado. Dessa vez, publicado na Annals Of Internal Medicine, o estudo "Hydroxychloroquine in Nonhospitalized Adults With Early: A Randomized Trial” foi desenvolvido nos Estados Unidos e no Canadá (SKIPPER et al., 2020).

Randomizado, duplo-cego, prospectivo, o estudo teve 6924 pessoas inscritas através de pesquisas na internet. Entretanto, dessa população, a amostra foi delimitada em 491 pessoas. Houve ainda mais um recorte, visto que das 491, 423 pessoas realmente contribuíram para o levantamento dos dados. (SKIPPER et al., 2020). 
A amostra continha adultos de 40 estados dos Estados Unidos e de 3 províncias do Canadá, sintomáticos, não hospitalizados, com COVID-19 confirmado por laboratório ou com sintomas compatíveis com a doença após uma exposição de alto risco a uma pessoa com COVID-19, cujo diagnóstico foi confirmado por PCR nos últimos 14 dias anteriores ao estudo. A idade média dos pacientes que compuseram a amostra era de 40 anos (SKIPPER et al., 2020).

As variáveis estranhas, isto é, aquelas aptas a influenciarem o resultado do experimento, mas que não eram objeto do mesmo, como características pessoais (idade, peso, gênero) e demográficas, estado clínico, comorbidades, se fumante, se trabalhador de saúde, foram todas objeto de tentativa de controle de equilíbrio, de modo que incidissem da mesma maneira em todos os grupos (SKIPPER et al., 2020).

Pelo fato de ser randomizado, os participantes do estudo foram aleatoriamente distribuídos entre os grupos controle e experimental. 211 pacientes compuseram o grupo controle e 212, o grupo experimental. Durante 14 dias, o grupo controle recebeu placebo como tratamento. O grupo experimental recebeu $800 \mathrm{mg}$ de hidroxicloroquina uma vez, seguida de 600mg de 6 a 8 horas e, depois, 600mg diariamente por mais 4 dias, via oral (SKIPPER et al., 2020).

Foram obtidos como resultados dos experimentos os seguintes dados: a mudança na gravidade dos sintomas ao longo do período não diferiu entre os grupos controle e experimental (diferença na gravidade dos sintomas: relativa, 12\%; absoluta, -0,27 pontos [IC 95\%, -0,61 a 0,07 pontos]; $\mathrm{P}=0,117$ ); no final do período de 14 dias, $24 \%$ dos participantes do grupo experimental e 30\% do grupo controle ainda apresentaram sintomas; efeitos adversos da medicação ocorreram em $43 \%$ dos participantes do grupo experimental contra $22 \%$ do grupo controle; no grupo controle ocorreram 10 internações (2 não relacionadas ao COVID-19) e 1 óbito hospitalizado; no grupo experimental ocorreram 4 internações hospitalizadas (SKIPPER et al., 2020).

Concluiu-se que o tratamento composto pela manipulação da hidroxicloroquina não foi capaz de reduzir substancialmente a gravidade dos sintomas em pacientes diagnosticados com COVID-19 em estado leve e precoce (SKIPPER et al., 2020).

A partir dos resultados e das conclusões obtidas no estudo americano e canadense, controlado, randomizado, duplo-cego e com amostra robusta, a Sociedade Brasileira de Infectologia (SBI), filiada à Associação Médica Brasileira (AMB), emitiu, no dia 17 de julho, o Informe $n^{\circ} 16$, contendo atualização sobre a hidroxicloroquina no tratamento precoce da COVID-19 (SOCIEDADE BRASILEIRA DE INFECTOLOGIA, 2020). No Informe, a SBI orientou que a hidroxicloroquina fosse abandonada em qualquer fase do tratamento da COVID- 
19. Segundo a Sociedade, tal orientação acompanhou as que estão sendo dadas por todas as sociedades médicas científicas dos países desenvolvidos e pela própria Organização Mundial da Saúde (SOCIEDADE BRASILEIRA DE INFECTOLOGIA, 2020).

Recomendou-se, ainda, em caráter de urgência e necessidade, que os agentes públicos (estados, municípios e Ministério da Saúde) “reavaliem suas orientações de tratamento, não gastando dinheiro público em tratamentos que são comprovadamente ineficazes e que podem causar efeitos colaterais” e que o recurso público seja utilizado para a compra de medicamentos eficazes e seguros comprovadamente para pacientes com COVID-19 (SOCIEDADE BRASILEIRA DE INFECTOLOGIA, 2020, p. 2).

Na data de 23 de julho de 2020 foi publicado um estudo na The New England Journal of Medicine, dessa vez brasileiro e nomeado "Hydroxychloroquine with or without Azithromycin in Mild-to-Moderate Covid-19”. O estudo em questão objetivou produzir evidências científicas acerca da segurança e da eficácia dos tratamentos para COVID-19 compostos pelos medicamentos hidroxicloroquina e azitromicina (CAVALCANTI et al., 2020). Tratou-se de um estudo aprovado pela Agência Nacional de Vigilância Sanitária (ANVISA), randomizado, duplo-cego, prospectivo e com uma amostra de 665 pacientes maiores de 18 anos, hospitalizados com suspeita ou confirmação de COVID-19, sintomáticos há no máximo 14 dias. O delineamento experimental escolhido para esse estudo contou com três grupos, um controle e dois experimentais. A média de idade dos pacientes da amostra era de 50,3 anos, e esses estavam espalhados em 55 hospitais pelo país (CAVALCANTI et al., 2020).

As variáveis estranhas como características pessoais (idade, peso, gênero) e demográficas, medicamento usado antes do início do experimento e coexistência de comorbidades foram todas controladas e equilibradas, de modo que incidissem da mesma maneira em todos os grupos (CAVALCANTI et al., 2020).

O grupo controle continha 227 pacientes, que recebiam o tratamento padrão (glicocorticóides, imunomoduladores, antibióticos e antivirais). O primeiro grupo experimental continha 221 pessoas, que recebiam o tratamento padrão somado a hidroxicloroquina isolada (400mg, 2 vezes ao dia). O segundo grupo experimental com 217 pacientes recebeu como o tratamento padrão e a hidroxicloroquina (400mg, 2 vezes ao dia) associada a azitromicina (500g, 1 vez ao dia). As medicações foram aplicadas durante 7 dias, entretanto, o acompanhamento experimental durou duas semanas (CAVALCANTI et al., 2020).

Após 14 dias, chegou-se aos seguintes resultados: 68\% dos pacientes do grupo controle receberam alta; enquanto $64 \%$ do primeiro grupo experimental e $69 \%$ do segundo grupo 
experimental tiveram alta; 3 pacientes do grupo controle vieram a óbito, ao passo que 3 pacientes do primeiro grupo experimental e 2 pacientes do segundo grupo experimental faleceram (CAVALCANTI et al., 2020).

Após a apresentação dos resultados, os pesquisadores concluíram que não foram encontradas diferenças significativas após o experimento entre os pacientes que estavam no grupo controle, aqueles que estavam no primeiro grupo experimental e aqueles que estavam no segundo grupo experimental. Além disso os pacientes que receberam hidroxicloroquina isolada ou com azitromicina tiveram com maior frequência eventos de prolongamento do intervalo QT e elevação dos níveis de enzimas hepáticas em relação aos pacientes não receberam nenhum agente (CAVALCANTI et al., 2020).

Esse escorço reforça que as evidências iniciais eram inconsistentes e, portanto, insuficientes para fundamentar a política pública de incorporação da terapia em apreço pelo sistema público de saúde. Tivessem sido considerados os critérios legalmente firmados para essa política de saúde e os marcos teóricos adotados pelo próprio MS, a incorporação da terapêutica não teria, provavelmente, sido implementada, o que faz com que seja questionável a legitimidade das medidas adotadas em relação à cloroquina e à hidroxicloroquina.

\section{CONSIDERAÇÕES FINAIS}

Se o intento do trabalho é apreciar o papel da Medicina Baseada em Evidências científicas para a incorporação de terapêuticas pelo Sistema Único de Saúde (SUS), parece necessário apontar que os argumentos da autonomia do paciente para decidir sobre seu tratamento e da licitude da prescrição off label de medicamentos guardam pouca ou nenhuma relação com o debate atinente à política pública, no que toca aos parâmetros da lei específica de regência.

O aparato ao exercício de autonomia terapêutica do paciente é tão maior quanto mais conhecedor ele for das evidências, em seus diversos graus. Assim, o paciente pode edificar sua concepção particular de saúde, que é consonante com o livre desenvolvimento de sua personalidade. Em relação à prescrição off label de medicamentos, o médico deve agir com perícia no exercício da profissão, tendo em mente as evidências e articulando-as em favor do paciente, o que pode representar parâmetro de licitude e de reforço de conduta proba, isenta de culpa. Por lei, essas perspectivas não são, entretanto, critérios justificadores de políticas públicas de incorporação de tratamentos no SUS. 
Para a crítica das políticas públicas de amplo espectro de disponibilização generalizada de medicamentos no SUS, a robustez das evidências tende a assegurar a observância do interesse público. Lado outro, empregar evidências de baixa qualidade pode, eventualmente, macular a legalidade do ato administrativo correspondente.

A Medicina ou Saúde Baseada em Evidências, por meio dos seus instrumentos, como o Sistema GRADE, adotado pelo Ministério da Saúde, oferece (ou, pelo menos, deveria oferecer) substrato científico para a análise empreendida nesse trabalho.

O Sistema GRADE demonstra que os desenhos metodológicos de estudos clínicos são determinantes dos níveis de evidência oriundos desses trabalhos. Quanto mais sofisticado for o delineamento experimental, mais robusta é a evidência. Percebe-se que os graus mais elevados de evidência envolvem o controle de vieses, randomização, mascaramento e amostra representativa capaz de elevar o potencial de generalização.

Entretanto, é possível concluir que os estudos clínicos com cloroquina e com seu análogo hidroxicloroquina aludidos nas Notas Informativas $n^{\circ}$ 6/2020-DAF/SCTIE/MS e $n^{0}$ 9/2020-SE/GAB/SE/MS do Ministério da Saúde - que preveem o emprego dessas medicações no tratamento dos casos leves e graves da COVID-19 - são experimentos que apresentam falhas associadas a baixos graus de evidências científicas.

O referenciado estudo francês (The Méditerranée Infection University Hospital Institute in Marseille), além de ser não-randomizado e não mascarado, apresentou seus resultados com base em uma amostra consideravelmente pequena de 36 pacientes, sem controle de variáveis estranhas relevantes. O também já referenciado estudo chinês (Shanghai Public Health Clinical Center), ainda que randomizado, não foi mascarado e trabalhou com uma amostra insuficiente para viabilizar grau de generalização elevado, por ser composta de apenas 30 participantes. Não houve também o controle de variáveis estranhas. Especialmente no primeiro estudo, foi demonstrado suposto benefício da cloroquina ou da hidroxicloroquina no combate ao SARS$\mathrm{CoV}-2$.

Quando se analisam estudos com desenhos metodologicamente aprimorados, os resultados são inversamente proporcionais. Sobre os estudo com evidências mais robustas já explorados nesse ensaio, o norte-americano e canadense "Hydroxychloroquine in Nonhospitalized Adults With Early COVID-19: A Randomized Trial”, que foi randomizado, duplo-cego, com universo amostral de 423 pessoas e controle de variáveis confundidoras, demonstrou que os pacientes com COVID-19 que receberam hidroxicloroquina não tiveram benefício clínico em comparação com aqueles que receberam placebo. 
O mencionado estudo brasileiro "Hydroxychloroquine with or without Azithromycin in Mild-to-Moderate Covid-19”, randomizado, duplo-cego, com controle de variáveis estranhas e com o universo amostral de 665 pessoas, demonstrou que pacientes que trataram o SARS-CoV2 com hidroxilocloroquina não apresentaram benefício clínico, ainda que o medicamento tenha sido associado a azitromicina.

Essa trajetória na busca por evidências científicas de qualidade em relação aos medicamentos estudados revela a provável inconsistência das políticas públicas de saúde correlatas, quando considerados o papel da MBE e os critérios objetivos legalmente fixados.

Ao fim e ao cabo, não se trata, como dito, de realizar discussão apenas sobre a eficácia da cloroquina e hidroxicloroquina. O que se afere pela via desse estudo do caso da medicação em debate é que evidências científicas têm muito a contribuir para o substrato científico suficiente ao atendimento do critério objetivo legal para a incorporação de tratamentos pelo sistema público de saúde nacional.

Esses três recortes possíveis do problema merecem esses esclarecimentos, para que o contexto da pandemia da COVID-19 não turve os debates sobre o direito à saúde e não permita que, em nome da autodeterminação terapêutica, sejam incorporados ao sistema de saúde e ofertados à população novos fármacos, novos produtos, novos procedimentos, novas tecnologias, novos protocolos clínicos e novas diretrizes terapêuticas cuja segurança, eficácia e acurácia estejam lastreadas em evidências com baixo grau de qualidade.

\section{REFERÊNCIAS}

AGÊNCIA NACIONAL DE VIGILÂNCIA SANITÁRIA. Resolução RDC n. 09, de 20 fev. 2015. Dispõe sobre o Regulamento para a realização de ensaios clínicos com medicamentos no Brasil. Brasília, Diário Oficial da União, 03 mar. 2015. Disponível em: http://portal.anvisa.gov.br/documents/10181/3503972/RDC_09_2015_COMP.pdf/e26e9a449cf4-4b30-95bc-feb39e1bacc6. Acesso em: 30 mar. 2020.

AGÊNCIA NACIONAL DE VIGILÂNCIA SANITÁRIA. Resolução RDC n. 200, de 26 dez. 2017. Dispõe sobre os critérios para a concessão e renovação do registro de medicamentos com princípios ativos sintéticos e semissintéticos, classificados como novos, genéricos e similares, e dá outras providências. Brasília, Diário Oficial da União, 28 dez. 2017, republicado em 29 jan. 2018.2 Disponível em: http://portal.anvisa.gov.br/documents/10181/3836387/RDC_200_2017_COMP.pdf/3b8c3b31 -24cb-4951-a2d8-8e6e2a48702f. Acesso em: 06 jun. 2020.

BRASIL. Decreto n. 20.931, de 11 jan. 1932. Regula e fiscaliza o exercício da medicina, da odontologia, da medicina veterinária e das profissões de farmacêutico, parteira e enfermeira, no Brasil, e estabelece penas. Diário Oficial da União, Rio de Janeiro, 31 dez. 1932. Disponível 
em: https://www.planalto.gov.br/ccivil _03/decreto/1930-1949/ d20931.htm. Acesso em: 06 jun. 2020;

BRASIL. Lei n. 13.979, de 6 de fev. 2020. Dispõe sobre as medidas para enfrentamento da emergência de saúde pública de importância internacional decorrente do coronavírus responsável pelo surto de 2019. Diário Oficial da República Federativa do Brasil, Brasília, DF, 7 fev. 2020. Disponível em: http://www.planalto.gov.br/ccivil_03/_ato20192022/2020/lei/l13979.htm. Acesso em: 23 jul. 2020.

BRASIL. Lei Federal n. 6.360, de 23 setembro 1976. Dispõe sobre a vigilância sanitária a que ficam sujeitos os medicamentos, as drogas, os insumos farmacêuticos e correlatos, cosméticos, saneantes e outros produtos, e dá outras providências. Diário Oficial da União, Brasília, 24 set. 1976. Disponível em: http://www.planalto.gov.br/ccivil_03/leis/l6360.htm. Acesso em: 6 jun. 2020.

BRASIL. Lei Federal n. 8.080, de 19 set. 1990. Dispõe sobre as condições para a promoção, proteção e recuperação da saúde, a organização e o funcionamento dos serviços correspondentes e dá outras providências. Diário Oficial da União, Brasília, 20 set. 1990. Alterada por: BRASIL. Lei Federal n. 12.401, de 28 abr. 2011. Diário Oficial da União, Brasília, 29 abr. 2011. Disponível em: http://www.planalto.gov.br/ccivil/leis/l8080.htm. Acesso em: 06 jun. 2020.

BRASIL. Ministério da Saúde. Diretrizes para diagnóstico e tratamento da COVID-19. Brasília: Ministério da Saúde, 06 abr. 2020. Disponível em: https://portalarquivos.saude.gov.br/images/pdf/2020/April/07/ddt-covid-19.pdf. Acesso em: 06 jun. 2020.

BRASIL. Ministério da Saúde. Secretaria de Ciência, Tecnologia, Inovação e Insumos Estratégicos em Saúde. Departamento de Assistência Farmacêutica e Insumos Estratégicos. Nota Informativa $n^{0}$ 6/2020-DAF/SCTIE/MS. Atualiza informações sobre o Uso da Cloroquina como terapia adjuvante no tratamento de formas graves do COVID-19, ficando revogada a Nota Informativa $n^{\circ}$ 5/2020-DAF/SCTIE/MS, datada de 27 de março de 2020. Brasília: Ministério da Saúde, 2020.

BRASIL. Ministério da Saúde. Secretaria Executiva. Gabinete da Secretaria Executiva. Nota Informativa no 9/2020-SE/GAB/SE/MS. Orientações para manuseio medicamentoso precoce de pacientes com diagnóstico da COVID-19. Brasília: Ministério da Saúde, 2020.

CAVALCANTI et al. Hydroxychloroquine with or without Azithromycin in Mild-to-Moderate Covid-19. The New England Journal of Medicine, p. 1-12 2020. DOI: 10.1056/NEJMoa2019014.

em: https://www.nejm.org/doi/full/10.1056/NEJMoa2019014?query=featured_home. Acesso em: 25 jul. 2020.

CHEN, Jun et al. A pilot study of hydroxychloroquine in treatment of patients with moderate COVID-19. Journal of ZheJiang University (Medical Sciences), vol. 49, Issue 2, p. 215-219, 2020. DOI: 10.3785/j.issn.1008-9292.2020.03.03. Disponível em: http://www.zjujournals.com/med/EN/10.3785/j.issn.1008-9292.2020.03.03. Acesso em: 25 jul. 2020. 
CONSELHO FEDERAL DE MEDICINA. Código de ética médica: resolução CFM n ${ }^{\circ} 2.217$, de 1 de novembro 2018. Aprova o Código de Ética Médica. Conselho Federal de Medicina, Brasília: 2018. Diário Oficial da União: 01 nov. 2018. Disponível em: https://www.anamt.org.br/portal/wpcontent/uploads/2018/11/resolucao_cfm_n_22172018.pdf. Acesso em: 6 jun. 2020.

CONSELHO FEDERAL DE MEDICINA. Processo-Consulta CFM n $\mathbf{n}^{\mathbf{0}} \mathbf{8} / \mathbf{2 0 2 0}$ - Parecer CFM no 4/2020, de 16 de abril de 2020. Tratamento de pacientes portadores de COVID-19 com cloroquina e hidroxicloroquina. Considerar o uso da cloroquina e hidroxicloroquina, em condições excepcionais, para o tratamento da COVID-19. Brasília: Conselho Federal de Medicina, 2020.

EL DIB, Regina. Medicina Baseada em Evidências. In: EL DIB, Regina (Org.). Guia Prático de Medicina Baseada em Evidências. São Paulo: Cultura Acadêmica, 2014.

FERREIRA, Aurélio Buarque de Hollanda. Novo Dicionário da Língua Portuguesa. Curitiba: Positivo Informática, 2004. Versão 5.0. CD.

GAUTRET, Philippe et al. Hydroxychloroquine and azithromycin as a treatment of COVID19: results of an open-label non-randomized clinical trial. International Journal of Antimicrobial Agents, vol. 56, Issue 1, p. 1-6, 2020. DOI: 10.1016/j.ijantimicag.2020.105949. Disponível em: https://www.sciencedirect.com/science/article/pii/S0924857920300996. Acesso em: 25 jul. 2020.

GOMES, Marleide da Mota; KALE, Pauline Lorena. Qualidade das evidências: desenhos de pesquisa. In: GOMES, Marleide da Mota. Medicina baseada em evidências: princípios e práticas. Rio de Janeiro: Reichmann \& Affonso Editora, 2001.

LIMA, Taisa Maria Macena de; BERALDO, Leonardo de Faria; NOGUEIRA, Roberto Henrique Pôrto. Reflexões sobre a responsabilidade civil do médico em tempos de pandemia da COVID-19. In: CRUZ: Álvaro Ricardo de Souza; PEREIRA, Maria Fernanda Pires de Carvalho (Org.). A pandemia e seus reflexos jurídico. Belo Horizonte: Arraes Editores, 2020, p. 211-224.

MEHRA, Mandeep R.; DESAI, Sapan S.; RUCHITZKA, Frank; PATEL, Amil N. RETRACTED: Hydroxychloroquine or chloroquine with or without a macrolide for treatment of COVID-19: a multinational registry analysis. The Lancet, p. 1-10, 2020. DOI: 10.1016/S0140-6736(20)31180-6. https://www.thelancet.com/journals/lancet/article/PIIS0140-6736(20)31180-6/fulltext. Acesso em: 25 jul. 2020.

MEHRA, Mandeep R.; RUCHITZKA, Frank; PATEL, Amil N. RetractionHydroxychloroquine or chloroquine with or without a macrolide for treatment of COVID-19: a multinational registry analysis; The Lancet, vol. 395, Issue: 10240, 2020. DOI: 10.1016/S0140-6736(20)31324-6. Disponível em: https://www.thelancet.com/journals/lancet/article/PIIS0140-6736(20)31324-6/fulltext. Acesso em: 25 jul. 2020.

MINISTÉRIO DA SAÚDE. Secretaria de Ciência, Tecnologia e Insumos Estratégicos. Departamento de Ciência e Tecnologia. Diretrizes metodológicas: Sistema GRADE - 
Manual de graduação da qualidade da evidência e força de recomendação para tomada de decisão em saúde. Brasília: Ministério da Saúde, 2014. Disponível em: https://bvsms.saude.gov.br/bvs/publicacoes/diretrizes_metodologicas_sistema_grade.pdf. Acesso em: 25 jul. 2020.

NOGUEIRA, Roberto Henrique Pôrto; GODOI, Nayder Rommel de Araújo. Objeção de consciência e a recusa do médico para a prescrição de cloroquina ou hidroxicloroquina em associação com azitromicina no tratamento da COVID-19. Emporio do direito, 2020. Disponível em: https://emporiododireito.com.br/leitura/objecao-de-consciencia-e-a-recusa-domedico-para-a-prescricao-de-cloroquina-ou-hidroxicloroquina-em-associac-a-o-comazitromicina-no-tratamento-da-covid-19. Acesso em: 23 jul. 2020.

NOGUEIRA, Roberto Porto Henrique. Prescrição off label de medicamentos, ilicitude e responsabilidade civil do médico. Belo Horizonte: Editora PUC Minas, 2017.

NOGUEIRA, Roberto Henrique Pôrto. Responsabilidade civil do médico na prescrição off label de medicamentos para a COVID-19. In: MONTEIRO FILHO, Carlos Edison do Rêgo; ROSENVALD, Nelson; DENSA, Roberta (Org.). Coronavírus e responsabilidade civil: impactos contratuais e extracontratuais. Indaiatuba: Editora Foco, 2020, p. 283-292.

ORGANIZAÇÃO PAN-AMERICANA DA SAÚDE. Atualização Dinâmica e Contínua de Potenciais Terapias para COVID-19: resumo de revisões sistemáticas rápidas, 22 de abril de 2020. Organização Mundial da Saúde, 2020. Disponível em: https://iris.paho.org/handle/10665.2/52173. Acesso em: 25 jul. 2020.

SELLTIZ, Claire; WRIGHTSMAN, Lawrence Samuel; COOK, Stuart Wellford; KIDDER, Louise H. (org.). Métodos de pesquisa nas relações sociais. São Paulo: EPU, 1987.

SKIPPER et al. Hydroxychloroquine in Nonhospitalized Adults With Early COVID-19: A Randomized Trial, Annals of Internal Medicina, 2020. DOI: https://doi.org/10.7326/M204207. Disponível em: https://www.acpjournals.org/doi/10.7326/M20-4207. Acesso em: 25 jul. 2020.

SOCIEDADE BRASILEIRA DE INFECTOLOGIA. Informe $\mathbf{n}^{\circ} 16$ Da Sociedade Brasileira De Infectologia Sobre: Atualização Sobre A Hidroxicloroquina No Tratamento Precoce Da Covid-19, de 17 de julho de 2020. São Paulo: Sociedade Brasileira de Infectologia, 2020.

VILLAS BOAS, Paulo José Fortes; DO VALLE, Adriana Polachini. Níveis de evidência e grau de recomendação. In: EL DIB, Regina (Org.). Guia Prático de Medicina Baseada em Evidências. São Paulo: Cultura Acadêmica, 2014.

WANG et al. A review of the 2019 Novel Coronavirus (COVID-19) based on current evidence. International Journal of Antimicrobial Agents - In Press 17 March 2020 - DOI: 10.1016/j.ijantimicag.2020.105948. Disponível em: https://www.sciencedirect.com/science/article/pii/S0924857920300984. Acesso em: 9 abr. 2020. 